\title{
Deutsch als Fremdsprache
}

\section{Zeitschrift zur Theorie und Praxis des Deutschunterrichts für Ausländer}

\section{INHALTSVERZEICHNIS}

Johannes Wenzel

10 Jahre interDaF

Barthold C. Witte

Die auswärtige Kultur- und Sprachpolitik des vereinten Deutschland. Erwartungen, Chancen, Probleme

Mathilde Hennig

„Die hat doch Performanzschwierigkeiten." Performanzhypothese und Kompetenz(en)gegenthese

Eva Breindl/Maria Thurmair

Wie viele Grammatiken verträgt der Lerner? Zum Stellenwert einer „Grammatik der gesprochenen Sprache" (nicht nur) für Deutsch als Fremdsprache

Jörg Roche

Plädoyer für ein theoriebasiertes Verfahren von Software-Design und Software-Evaluation

Nicole Schumacher

Perspektiven für die Vermittlung von Tempusbedeutungen im Rahmen von $\mathrm{DaF}$

Rezensionen

Helmut Brammerts/Karin Kleppin (Hg.): Selbst gesteuertes Sprachenlernen im Tandem. Ein Handbuch; Albert Raasch

Josef Leisen (Hg.): Methoden-Handbuch. Deutschsprachiger Fachunterricht (DFU); Horst Raabe

Ulla Fix u. a.: Textlinguistik und Stilistik für Einsteiger. Ein Lehr- und Arbeitsbuch; Lutz Kuntzsch

Jörn Albrecht: Europäischer Strukturalismus. Ein forschungsgeschichtlicher Überblick. 2. Aufl.; Gerhard Helbig

Claudio Di Meola: Die Grammatikalisierung deutscher Präpositionen; Jochen Schröder

94 Suang-Jing Pong: Konjunktionen und konjunktionale Funktion; Gerhard Helbig

104

Schreibhinweise für Autoren

Diskussion von Lehrmaterialien

Christian Krekeler

Autorenverzeichnis

Wirtschaftsrecht im DaF-Fachsprachenunterricht. $\mathrm{Zu}$ einem neuen Lehrbuch 


\section{Plädoyer für ein theoriebasiertes Verfahren von Software-Design und Software-Evaluation*}

\section{Einleitung}

Als Lehrerin oder Lehrer muss man sich heutzutage immer wieder - und ,immer öfter" - fragen, ob es denn irgendwelche elektronischen Programme gibt, die den Unterricht erleichtern und vielleicht auch interessanter und ergiebiger machen könnten. Die Suche danach endet allzu schnell in völliger Verwirrung oder Ratlosigkeit oder man lässt sich bei deren Lösung der Einfachheit halber von Erfahrungsberichten anderer Kollegen leiten - Erfahrungen, die sich dann aber leider allzu oft als nicht replizierbar erweisen. Um diesem Zustand Abhilfe zu verschaffen finden sich in jüngerer Zeit zunehmend Bestrebungen, Sprachlernsoftware systematisch $\mathrm{zu}$ erfassen und vergleichbar $\mathrm{zu}$ machen. Den beabsichtigten Erfolg haben diese Versuche allerdings bisher nicht gebracht. Die Liste der Kriterien wächst dabei ins Uferlose. Sie beginnt bei den globalen Bewertungen in Computerzeitschriften und endet bei mehrseitigen Kriteriensammlungen in Magisterarbeiten. Warum sind aber fast alle diese Listen ,aussagelos"? Die zwei grundsätzlichen Bewertungsverfahren, die eher empirische Bewertung durch Teilnehmer/Nutzer und die eher qualitativ-introspektive durch Experten, haben - so die These dieses Beitrags - in der Regel das große Manko, nicht theoriebasiert vorzugehen. Das heißt, sie haben wenige oder keine systematischen Bezüge zu Lerntheorien, Informationsverarbeitungstheorien, Spracherwerbstheorien, didaktischen Theorien oder linguistischen Modellen. Sie versammeln mehr oder weniger umfangreiche Kriterien (wenn sie nicht ohnehin völlig holistisch vorgehen), die aber ohne die nötigen Bezüge $\mathrm{zu}$ theoretischen Kontexten eigentlich zur Bedeutungslosigkeit verblassen. Ein Beispiel für ein derart verkürztes Evaluationsverfahren ist der Bewertungsbogen der Software-Fachtagung Fremdsprachen der e-nitiative Nordrhein-Westfalen vom 7. 3. 2001, der völlig unspezifiziert die Software von 1 bis 5 bewerten lässt.
Aber auch Spezifizierungen etwa nach einer Rubrik „didaktische Erläuterungen“, wie sie in manchen detaillierteren Listen gefunden werden können, geben zwar relevante Kriterien an, sagen damit aber noch nichts über die Eignung eines Programmes für bestimmte Lernzwecke aus. Bei solchen Listen scheint es meist, als sollte die zunehmende Fülle der Kriterien den Mangel an Aussagekraft kompensieren. Die von Thomé (1989) vorgestellte, aus 23 verschiedenen Katalogen zusammengestellte Liste von 324 Einzelkriterien, die nach kritischer Sichtung zu einer „Großen Prüfliste für Lernsoftware“ von immerhin noch 221 Kriterien komprimiert wurde, ist dafür ein anschauliches Beispiel. Die Folge solcher Verlistungen der Software ist eine unübersichtliche Menge von wenig kontextualisierten Einzelkriterien, deren Gewichtung und Bewertung dann doch subjektiver Einschätzung, d. h. dem Gutdünken, überlassen bleibt (vgl. Doll 1987: 58).

Bewertungen sind somit nicht replizierbar, nutzen also höchstens dem einzelnen Lehrer, der sie erstellt. Sie können darüber hinaus nicht als Grundlage für die Erarbeitung allgemein gültiger didaktischer Verfahren oder im Sinne formativer Evaluation - als Leitfaden für Softwareentwickler verwendet werden. Diese Feststellung wird von einer kürzlich durchgeführten Untersuchung gängiger Evaluationsverfahren untermauert (vgl. Reeder et al. 2001). ${ }^{\prime}$ Sie erbrachte zumindest im englischsprachigen Bereich keine Belege für formative Evaluationsverfahren von Sprachlernsoftware, die die Lernziele und die dahin

\footnotetext{
* Ich danke Stephan Schlickau für zahlreiche Hinweise und Diskussionen, die in diesen Beitrag eingeflossen sind.

1 Ausgenommen davon ist zu einem gewissen Grad lediglich die beobachtende Untersuchung der Lernprozesse und -strategien von Murray (1999) zu dem MultimediaProgramm „A la rencontre de Philippe“.
} 
führenden Lernprozesse systematisch untersuchen würden. ${ }^{1}$

In seinem „Methodological framework for CALL courseware development" versucht Hubbard (1992) immerhin eine operationale Systematik von relevanten Kriterien zu entwickeln. Diese sieht unter Bezug auf die Klassifikation von Richards/Rodgers (1986) eine Dreiteilung in die Kriterienbündel ,approach“, "design" und "procedure" vor (vgl. auch Tschirner 1995). Unter ,approach“ fasst er dabei linguistische, methodische und lernpsychologische „Annahmen“, aus denen sich unter Berücksichtigung der Eigenschaften des „Computer Delivery Systems" entsprechende Designkriterien ergeben. In das Design fließen schließlich vor allem die Lernervariablen und die Vorgaben des Lehrplans ein. Zusammen mit den Verfahrensvariablen (zum Beispiel Bedienungsoptionen, Bewertung von Lernerantworten, Hilfsfunktionen und Programm-

\footnotetext{
I Vgl. hierzu auch die theoretischen Darlegungen des Aufrufes zur Diskussion von Rösler/Tschirner (2002).

Dass es auch bei den gedruckten Lehrmaterialien gar nicht so viel besser aussieht, zeigen verschiedene Versuche standardisierte und aussagekräftige Verfahren der Lehrwerkevaluation zu erarbeiten: das „Mannheimer Gutachten“"von 1977 und 1979 (Engel et al. 1979), das „Gutachten zu ausgewählten Lehrwerken. Deutsch für ausländische Arbeiter" (Barkowski et al. 1980), die verschiedenen Arbeiten zur Lehrwerkanalyse des GoetheInstituts, wie z. B. der „Stockholmer Kriterienkatalog“ von 1990 (Goethe-Institut 1990; Kast/Neuner 1994) oder das "Gutachten des Sprachverbandes“ (Sprachverband 1994).

Und auch bei den Prüfungsstandards, den Zertifikaten oder Diplomen DaF, der DSH, TestDaF, dem Europäischen Referenzrahmen, den Proficiency Guidelines des American Council of Teachers of Foreign Languages (ACTFL), den Canadian Language Benchmarks, den ALTE-Niveaus oder anderen internationalen Standards sieht es nicht viel besser aus: Wo findet man dort, auch in den neuesten Referenzrahmen, etwa Bezüge zu Spracherwerbsmodellen, Psycholinguistik oder Psychologie? In Bezug auf die Erarbeitung systematischer Richtlinien für den Sprachunterricht verdienten daher $z$. B. die von Prokop an der University of Alberta mit Hilfe einer Arbeitsgruppe Anfang der 90er-Jahre entwickelten kanadischen Richtlinien mehr Beachtung, differenzieren sie doch die Anforderungen besonders im Bereich der interkulturellen Kompetenz.

2 Vgl. hierzu auch Tschirner (1997), der ähnlich argumentiert. $\mathrm{Zu}$ den Kriterien gibt es darüber hinaus eine Reihe weiterer, in der Regel spezifischerer Vorschläge. So behandelt Plass (1998: 35ff.) z. B. allein den Aspekt des Design-Modells für das Nutzer-Interface von Sprachlern-/-lehrsoftware unter vier verschiedenen Perspektiven: „craft approach“, „,enhanced software engineering approach", „technologist approach“ und ,cognitive approach".
}

Layout) wird die Softwareproduktion schließlich realisiert. Hubbard nähert sich der Problematik dabei insgesamt eher von der praktischen Ebene der Lehrplanentwicklung - des Curriculum-Designs -, das aus den drei Komponenten Entwicklung, Implementierung und Evaluation besteht. In seinem Modell sind diese drei Module miteinander verbunden und können ausdrücklich auf Unterrichtsprinzipien bezogen werden. Leider werden aber auch hier weder die Bezüge zu theoretischen Aspekten (Lerntheorien, linguistische Theorien, didaktische Ansätze etc.) noch das Zusammenspiel der verschiedenen Variablen untereinander hinreichend spezifiziert.

\section{Zur Klassifikation von Sprachlernsoftware}

Die Fokussierung und Gewichtung der Kriterien für Sprachlernsoftware kann auf verschiedene Art erfolgen, zum Beispiel nach Medium oder technischem System, nach Funktion, nach Unterrichtsmethode oder didaktischem Verfahren oder nach lerntheoretischen Bezügen. Nimmt man die Klassifikation nach dem Medium oder dem technischen Standard vor, so ergibt sich grob eine Differenzierung nach drei Generationen: DOS-Programme, Multimedia-Programme (mit den Varietäten Video-Disc, CD-ROM und Hypermedia) und Internet-Programme. Über die Eignung sagt eine solche rein technische Klassifikation nach Generationen zunächst aber nichts aus. ${ }^{2}$

Etwas aufschlussreicher ist dagegen die Klassifikation nach Kriterien der Funktionalität: nach tutorieller, situativer oder instrumentell-explorativ-referenzieller Ausrichtung. Unter tutoriellen Programmen versteht man stark gesteuerte Lehr- oder Wiederholungsprogramme, mit denen ein Lerner meist selbstständig grammatische, lexikalische oder phonetische Themen erarbeiten oder üben kann. In diesem Sinne ersetzen die elektronischen Programme traditionelle Übungsformen gedruckter Lehr- oder Übungsmaterialien (Arbeitsbücher). Dabei geben sie aber (in der Regel) einfache Rückmeldung (meist nach der Richtig-falsch-Dichotomie) und bieten eine statistische Fehlerauswertung. Auch situativ ausgerichtete Programme weisen in der Regel ähnliche Übungssequenzen auf. Allerdings sind diese dann meist an ein kurzes Video oder eine Audioaufnahme angeschlossen. Wortschatz und Strukturen sind somit situiert. Die Situationen unterscheiden sich in Art und 
Intention jedoch wenig von Kassetten- oder Filmaufnahmen in älteren Medien. An der Oberfläche kann dabei leicht der Eindruck entstehen, es liege diesen Programmen ein kommunikativer Ansatz zu Grunde. In Wirklichkeit zeigen aber die Bearbeitungsverfahren, dass es sich häufig um neobehavioristische Modelle handelt. Selbst das kommunikative Prinzip der Authentizität der Materialien wird in den meisten Fällen nicht beachtet. Für die eigene Umsetzung (produktive Fertigkeiten) durch die Lerner haben die situativen Programme wenig Nutzen. Ihre Stärke liegt im Illustrationscharakter.

Die instrumentell-explorativ-referenziellen Programme betonen dagegen den authentischen, kommunikativen Nutzen elektronischer Werkzeuge bei der Umsetzung sprachhandlungsbedingter Funktionen. Zu diesen Werkzeugen gehören demnach Rechtschreibprüfungen, Thesauri, Webeditoren, Textverarbeitungsprogramme, E-Wörterbücher, E-Ressourcen, E-Fragebögen, E-Spiele und vieles mehr. Diese Programme erlauben den Lernern nicht nur Strukturen zu üben, sondern weit darüber hinaus kreativ und konstruktiv mit Sprache umzugehen. So kann zum Beispiel, wie bei dem Programm „Edubba“, eine Lernerin in der Rolle einer Redakteurin beim Verfassen von Zeitungsartikeln auf eine Reihe von Schreibund Recherche-Werkzeugen zurückgreifen, die gleichzeitig korrekte Strukturen vermitteln und helfen, den Wortschatz zu erweitern.

Bei einer funktionalen Einteilung lässt sich zumindest eine gewisse didaktische Kontextualisierung erkennen. Allerdings kann so nach wie vor nicht überzeugend begründet werden, warum eine bestimmte Funktion in einem bestimmten Programm gewählt worden ist bzw. gewählt werden sollte. Nach Funktionen klassifizierte Software ist im Prinzip nicht an ein bestimmtes Medium gebunden. Es bestehen aber bestimmte Affinitäten zwischen Funktion, didaktischem Ansatz und Wahl des Mediums, die allerdings bisher nur in dem theoretischen Konzept der Medienadäquatheit (vgl. Roche 2000), aber noch zu wenig in der konkreten Entwicklung von Software ihren Niederschlag gefunden haben. Tutorielle Systeme zum Beispiel eignen sich durchaus für geschlossene Programme und Medien und wurden daher auch vorwiegend in der Anfangsphase des „Computer Assisted Language Learning“ (CALL) eingesetzt. Die DOS-Um- gebung bot dafür ausreichende Realisierungsmöglichkeiten, selbst bei teilfarbigen und mit einfachen Animationen versehenen Tutorienprogrammen wie „TUCO“. Allerdings findet man auch heute noch ganze Tutorien (Grammatiktrainer, Vokabeltrainer, Ausspracheprogramme) in Multimedia oder Internet-Umgebungen eingebettet, ohne dass diese wesentlich über die Leistungen von DOS-Programmen hinausgingen und die Möglichkeiten der neueren Medien medienadäquat nutzen würden.

Bei den situativen Programmen besteht eine Affinität zu Multimedia-Programmen, die es zum ersten Mal ermöglichten, Kommunikationssituationen in kleinen Filmkonserven zu präsentieren. Die Funktion dieser situativen Sprachpräsentation besteht im Wesentlichen darin, den Lernern (pseudo-)authentische Sprache nicht nur im Schrifttext, sondern gleichzeitig auditiv und visuell als Input zur Verfügung zu stellen (zum Beispiel die CD-ROM-Programme „Einfach Toll“ oder „Business Challenges“). Dass dieses Bestreben - trotz vordergründiger Parallelen zu einem wichtigen Parameter des kommunikativen Sprachunterrichts - weniger theoretisch als animationistisch motiviert ist, zeigen die diesen Programmen in der Regel angegliederten, wenig nach den Prinzipien kommunikativer Didaktik ausgerichteten Aufgaben und Lehr-/Lernverfahren.

Instrumentell-explorativ-referenziell ausgerichtete Software hat ihre Hauptfunktion in der Unterrichtsbegleitung. Sie will autonomes Lernen ermöglichen und hat daher eher eine Affinität zu explorativ und im weitesten Sinne konstruktivistisch geprägten Lerntheorien. Allerdings berücksichtigt sie in der Regel noch zu wenig, dass die sprachlichen und interkulturellen Kompetenzen der anvisierten Lernergruppen häufig kein ausreichendes Niveau für einen erfolgreichen Zugang zu den Materialien darstellen und dass die Materialien die Lerner daher (oft auch schlicht wegen mangelnder Strukturiertheit) überfordern. ${ }^{1}$

Instrumentell-explorativ-referenzielle Programme lassen sich wegen der Möglichkeiten der prinzipiellen Veränderbarkeit und Offenheit (Linksammlungen, Archive) besonders gut

\footnotetext{
${ }^{1}$ Mit den Aspekten der interkulturellen Sprachdidaktik setzt sich Roche (2001) intensiv auseinander. Schlickau (2001) stellt eine aufschlussreiche Studie zu der Problematik interkultureller Kommunikation über die elektronischen Medien im Fremdsprachenunterricht dar.
} 
online realisieren. Einzelne Referenzmodule wie Wörterbücher und Grammatiken werden aber schon seit längerem auch in den geschlossenen Medien realisiert (veralten aber schneller). Umgekehrt wird das Flexibilitätspotenzial der offenen Medien häufig noch nicht von den Programmen ausgenutzt. Auch sie betonen weiterhin die rezeptiven Fertigkeiten des Sprachenlernens. Erst mit der fortschreitenden Entwicklung der Online-Kommunikationsmedien beginnt auch die Nutzbarmachung der generellen Offenheit der Online-Medien für didaktische Zwecke (zum Beispiel bei der Entwicklung von elektronischen Lernplattformen).

Unabhängig von den genannten Kriterien ließe sich Sprachlernsoftware auch danach klassifizieren, für welche Sprachlehrmethoden sie sich eignet, welche Fertigkeiten sie vermitteln will beziehungsweise welchen didaktischen Ansatz sie verfolgt, also z. B., ob sie grammatische Strukturen in den Mittelpunkt stellt, sich in Pattern-Drill-Übungen erschöpft, kommunikative Fertigkeiten anstrebt oder gar interkulturelle Fertigkeiten vermitteln kann.

Eine Zuordnung der Methoden zu den zuvor genannten Mediengenerationen lässt sich ebenfalls nur grob vornehmen, auch wenn es bestimmte Affinitäten zwischen GrammatikÜbersetzungsmethode und DOS-Programmen oder zwischen AV und Multimedia-Programmen gibt. Besonders diffus ist das Bild aber in neueren Programmen, bei denen ein undifferenzierter und unreflektierter Methodenpluralismus herrscht. Festzuhalten bleibt im Übrigen, dass der mediale Fortschritt ganz allgemein zunächst zu didaktischen Rückschritten geführt hat, und zwar um mehrere Generationen (vgl. Roche 2002).

\section{Lerntheorien und Fremdsprachenerwerb}

Behavioristische, kognitivistische und konstruktivistische Theorien üben (in der Regel über die Methoden und in noch genauer zu spezifi-

\footnotetext{
' Issing (1997) und Baumgartner (1997) stellen die verschiedenen instruktionistischen und konstruktivistischen Ansätze übersichtlich dar.

2 Reinfried (1999) stellt die Annahmen des radikalen Konstruktivismus in Bezug auf die Sprachdidaktik ausführlich dar und setzt sich mit ihnen kritisch auseinander. Er plädiert dagegen für einen kritisch-realistischen Konstruktivismus.

${ }^{3}$ Papert selbst nennt als anschauliche Beispiele den Bau von Sandburgen am Strand oder die Schaffung komplexer Theorien des Universums.
}

zierender Weise) Einflüsse auf den Unterricht und die Gestaltung der Lehrwerke aus. Fest steht allerdings, dass sie dies mit unterschiedlicher Stringenz und Konsequenz tun. Programmatisch-exklusiv ist bisher eigentlich nur versucht worden, behavioristische Theorien in Lehrmaterialien umzusetzen, und zwar bekanntlich mit zweifelhaftem Erfolg. Dennoch finden sich auch in neuerer Software massenhaft rein behavioristisch geprägte Verfahrensweisen (zum Beispiel in der mehrsprachigen „Rosetta Stone"-Serie, in dem Englisch-Programm „TMX“, in der mehrsprachigen CDROM-Reihe „Wer ist Oscar Lake?" oder in dem 1999 in Russland entstandenen Programm „What to say and how to behave in Great Britain"). Dabei wird allerdings die jeweilige Lerntheorie selbst wenig - wenn überhaupt reflektiert. Häufig werden vielmehr naive Praktiken (wie sie den Autoren und Entwicklern vielleicht aus deren eigenem Sprachunterricht bekannt sind) schlichtweg imitiert oder anderweitig unreflektiert übernommen.

Da hier nicht näher auf die verschiedenen lerntheoretischen Ansätze eingegangen werden kann, sei nur auf einige relevante Literatur hingewiesen. Zum Kognitivismus äußern sich besonders Strittmatter/Mauel (1997), den kognitivistischen Programmtyp illustrieren die Lesekurse „Reading German“ oder „Reading Business Japanese".' Zu konstruktivistischen Ansätzen nehmen unter anderem Reinfried (1999), Wolff (1994) und Mayer (1997) in seiner "Generative Theory of Multimedia Learning“" Stellung. Mit der Bedeutung des Konstruktivismus für den Fremdsprachenunterricht haben sich im deutschsprachigen Raum auch Wendt (1996) und in Bezug auf die Nutzung der Medien besonders Rüschoff (1999) und Wolff (1996) auseinander gesetzt. ${ }^{2}$

Der Konstruktionismus, wie er von Papert (1980) entwickelt wurde, unterscheidet sich vom Konstruktivismus im Grunde vor allem durch die Intensität der Konkretisation. Es geht dem Konstruktionismus nämlich um die Schaffung konkreter Objekte im öffentlichen Raum $^{3}$ (Fischhaber 2002; Beers 2001; Rüschoff 1999; Goldman-Segall 1998; als Programmtyp z. B. „Berliner Sehen“, „A la rencontre de Philippe" oder "WebConstellations").

Um die Stärken instruktionistisch oder konstruktivistisch geprägter Verfahren gleichermaßen auszunutzen, sind in letzter Zeit verstärkt hybride Formen von Lernverfahren 
entwickelt bzw. wiederentdeckt worden. Dieser „moderate Konstruktivismus“ wird im Softwarebereich häufig unter dem Oberbegriff ,instruktionales Design der zweiten Generation" zusammengefasst. Seine Verfahren fördern das Lernen in komplexen und kontextualisierten Problemräumen, wobei allerdings der Erwerb hierfür wichtiger elaborierter kognitiver Strukturen mehr oder minder stark durch Instruktion gefördert werden kann. Der erkenntnistheoretische Relativismus des radikalen Konstruktivismus wird in diesem instruktionalen Design in seiner Absolutheit jedoch zurückgewiesen. Als mediale Realisierung hierfür bieten sich offene Lehr-/Lernsysteme mit tutoriellen Komponenten an (vgl. Strittmatter/Mauel 1997; als Programmtyp z. B. „Hollywood Theatrix“).

\section{Parameter eines theoriebasierten Evaluierungsmodells}

Die zum Teil polarisierte Diskussion der Vorund Nachteile der theoretischen Ansätze kann und muss hier nicht aufgenommen werden, weil die Absolutheitsansprüche der Theorien bisher ohnehin nicht begründet sind (vgl. auch Mitschian 2000). Die Ansätze im Bereich des instruktionalen Designs der zweiten Generation zeigen vielmehr, dass verschiedene theoretische Ansätze zu erfolgreichem Lernen führen können, häufig in einer neuen Art von Synthese. Die wesentliche Aufgabe der Didaktik wäre es, dabei zu erklären und zu erproben, welche theoretisch begründeten Verfahren bei welcher Zielsetzung und in welchen Lernkontexten die effizientesten sind. Somit könnte sie nicht nur wesentlich zu einer theoretisch fundierten Praxis des Fremdsprachenunterrichts beitragen, sondern gleichzeitig wichtige Beiträge für eine Modifizierung der idealisierten Lerntheorien liefern. Ein solches ,aufgeklärtes“ Verständnis von Didaktik lässt sich für Zwecke des Designs und der Evaluation von Software in einem mehrstufigen Modell (idealtypisch) abbilden (vgl. Abb. 1, S. 99). ${ }^{\prime}$

Grundsätzlich sind die Bedingungen des Lerners und des Lernens zu berücksichtigen, also die spezifischen Lernervariablen einer Lerngruppe oder eines Lerners und die allgemeinen (kognitiven) Lernuniversalien, die die Informationsverarbeitung und den Sprachund Wissenserwerb steuern. Diese Lernerdispositionen stellen Ausgangspunkt und Grund- lage des gesamten Lernkontextes dar. Sie bestimmen damit auch, ob und wie die Lernziele in geeigneter Weise realisiert werden können. Der Bereich der Lernziele selbst ist in Richtlernziele, Groblernziele und Feinlernziele unterteilt.

Die Richtlernziele betreffen dabei allgemeine Kompetenzen, wie sie beispielsweise auf Grund einer bestimmten Bildungspolitik, Bildungsideologie oder Bildungstradition entstehen. In unserem von kommunikativen Prämissen geprägten Bildungssystem sind das zum Beispiel kommunikative Kompetenzen, soziale Kompetenzen und interkulturelle Kompetenzen. Allerdings sind diese nicht, wie es auch die kommunikative Didaktik noch impliziert (und an Hand von Niveaustufen und anderen definierten Standards expliziert und praktiziert), als gegeben hinzunehmen, sondern vielmehr mit den Lernerdispositionen (interkulturell) in Einklang zu bringen. Diese Verhandelbarkeit sollte insbesondere auch für kulturbedingte Lerntraditionen gelten (vgl. Roche 2001). Aus der Bestimmung der dermaßen vermittelten Richtlernziele ergibt sich eine Präferenz für eine bestimmte Lerntheorie, die sowohl den Erwartungen der Lerner als auch den Anforderungen der Zielkultur zu entsprechen hat. Für die genannten Kompetenzen bieten sich solche lerntheoretischen Modelle an, die besonders auf soziales und autonomes Lernen abheben.

Auf der nachgeordneten Ebene der Groblernziele werden die angestrebten didaktischen Ansätze und Verfahren festgelegt, also zum Beispiel Fertigkeitsbereiche wie die Beherrschung der Grammatik, Lesekompetenz, Schreibkompetenz, das Erlernen bestimmter Diskursmuster und Ähnliches. Auch hier ist zu entscheiden, im Rahmen welches lerntheoretischen Ansatzes dies geschehen soll. Eine Übernahme des zuvor auf Richtlernzielebene determinierten lerntheoretischen Modells auf den didaktischen Ansatz ist dabei nicht zwingend vorgeschrieben. Dennoch können durchaus auch auf dieser Ebene sozial-autonome und konstruktivistisch geprägte Verfahren eingesetzt werden. Aus pragmatischen Interessen oder theoretischen Überlegungen heraus kann sich aber auch je nach den Gegebenheiten eine Präferenz für einen anderen didakti-

\footnotetext{
${ }^{1}$ Dieses Modell wird gemeinsam mit Stephan Schlickau erarbeitet und weiterentwickelt.
} 


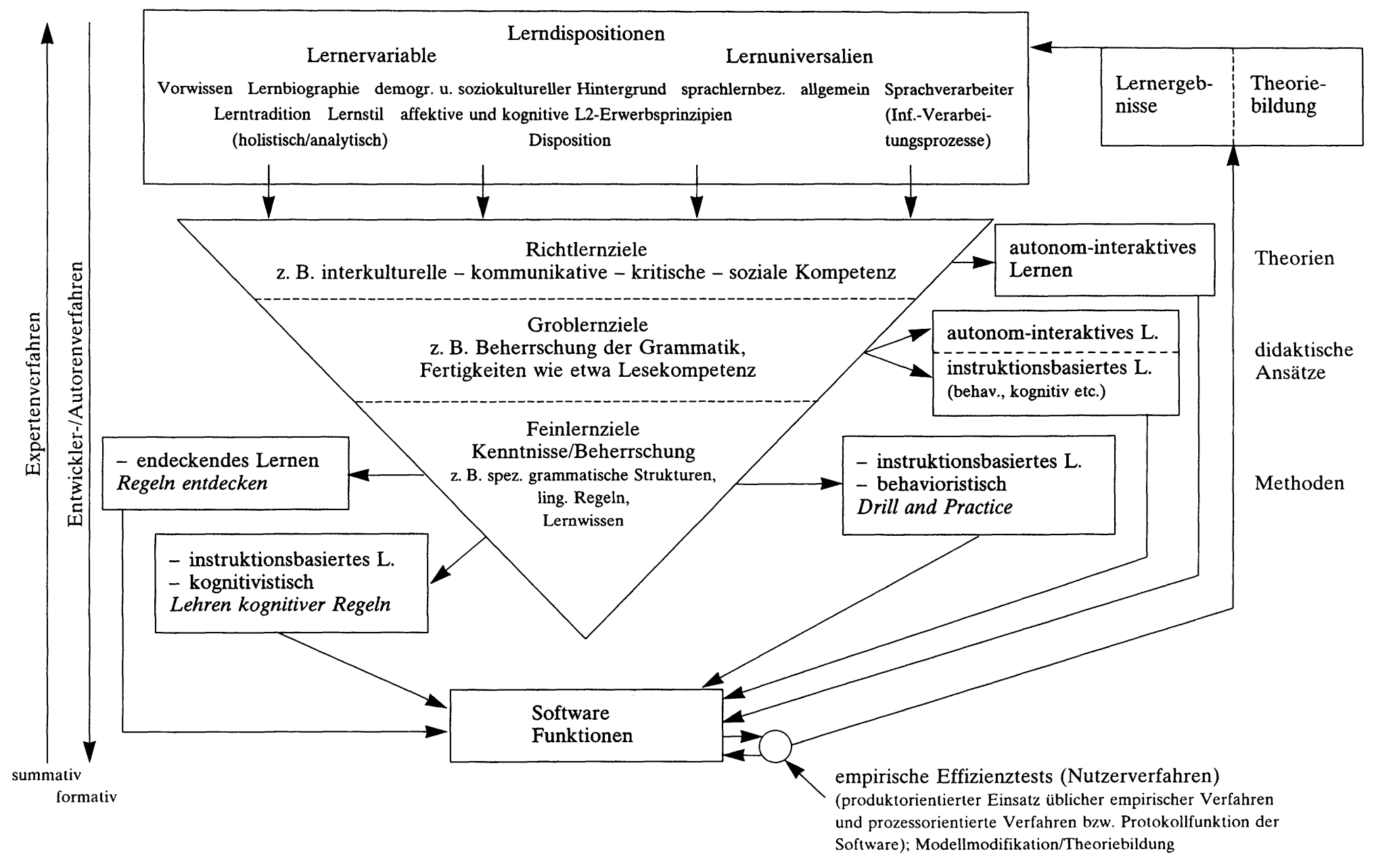


schen Ansatz ergeben. Ansonsten wäre eine Unterteilung in die drei verschiedenen Lernzielebenen im Prinzip auch überflüssig. Man kann also als Softwareentwickler durchaus einer konstruktivistischen Lerntheorie verbunden sein, aber für eine bestimmte Fertigkeitsebene wie die Grammatik eher einen kognitivistischen Ansatz wählen.

Innerhalb der dritten Lernzielebene schließlich ist $\mathrm{zu}$ bestimmen, welche Methoden bei der Umsetzung der Feinlernziele die geeignetsten sind. Zu den Feinlernzielen gehört beispielsweise die Kenntnis oder Beherrschung bestimmter sprachlicher Strukturen (wie die Beherrschung des Konjunktivs oder eines bestimmten Tempus oder die Kenntnis wichtiger touristischer Sprachkenntnisse), ein bestimmtes Lernwissen oder die Kenntnis bestimmter linguistischer Regeln. Je nach Lernziel und dem bereits zuvor genannten Lernkontext können sich auch auf dieser Ebene wieder verschiedene Optionen ergeben, die sich nicht notwendigerweise automatisch aus dem für die Entwicklung der Software gewählten lerntheoretischen Rahmen oder Ansatz ableiten lassen. Allerdings müssen sie miteinander verträglich sein. So bietet sich zum Beispiel ein induktives (Instruktions-)Verfahren bei der Grammatikvermittlung durchaus auch zur Operationalisierung von Groblernzielen im Rahmen einer kommunikativen Didaktik und eines konstruktivistischen Lernmodelles an. (Induktive Verfahren sind hier wegen der Lehrersteuerung grundsätzlich als instruktionistische Verfahren zu verstehen.) Selbst behavioristische PatternDrill-Methoden können - zum Beispiel bei reinen (eingebetteten) Automatisierungsroutinen - ihre Berechtigung innerhalb kommunikativkonstruktivistischer Verfahren und zur Verwirklichung autonom-interaktiver Richtlernziele bekommen. Mit einem dogmatisch vorgehenden und undifferenzierten audiovisuellen oder audiolingualen Ansatz, bei dem das Pattern Drill Inhalt und Ziel der Methode ist, haben derartig kontextualisierte Verfahren aber überhaupt nichts zu tun (vgl. Roche 2001; vgl. auch pragmatisch begründete Mischformen des ,instruktionalen Designs der zweiten Generation" wie „Cognitive Flexibility" von Spiro et al. 1991, "Cognitive Apprenticeship“ nach Collins et al. 1989, „Minnesota Adaptive Instructional System“ von Tennyson/Christensen 1988 und "Anchored Instruction“ der Cognition and Technology Group 1992).
Ein derart strukturiertes Modell hätte nicht nur den Vorteil, die medialen Produkte, die gezielt für das Sprachlehren und -lernen produziert werden, kontextualisiert und validierbar zu evaluieren. Es ließe sich im Grunde zur Feststellung der Eignung für Sprachlernzwecke auf jede Software anwenden. Dabei wird sich nachweisen und begründen lassen, dass oft gerade die Software, die eigentlich nicht für Sprachlehr-/-lernzwecke entwickelt wurde, für den Spracherwerb viel besser geeignet ist als so manches Sprachlehrprogramm. Zu denken ist dabei z. B. an die verschiedenen authentischen Werkzeuge virtuellen Kommunizierens und Arbeitens (von der einfachen SMS bis hin $\mathrm{zu}$ virtuellen Anamnesewerkzeugen der Mediziner), wie sie z. B. gezielt im studienbegleitenden Modul des neuen Online-Sprachprogramms „unideutsch.de "1 zum Einsatz kommen, aber auch die verschiedensten Simulationsspiele für alle möglichen fachlichen und spielerischen Zwecke (z. B. die „Hollywood Theatrix"-Serie für das Verfassen von englischsprachigen Cartoondrehbüchern oder geschichtliche Simulationsspiele wie „Fugger“ oder „Anno 1602/Anno 1503“). Auch in elaborierten (konstruktivistischen und konstruktionistischen Theorien verbundenen) Sprachlernprogrammen des fiktionalen oder dokumentarischen Typs, wie „A la rencontre de Philippe“, „Dans un quartier de Paris“ oder „Berliner Sehen“, wird im Übrigen auf ganz ähnliche Weise versucht die Authentizität sprachlicher Handlungen in den Unterricht zurückzubringen.

\section{Zur Operationalisierung von Evaluierungsverfahren}

Bei den Bewertungsverfahren von Sprachlehr-/-lernsoftware unterscheidet man allgemein zwischen formativer und summativer Evaluation. Die formative Evaluation bezeichnet die Erprobung und das Testen der Software während des Entwicklungsprozesses (in der Regel nach Abschluss der Hauptentwicklungsphase). Die summative Evaluation bezeichnet

\footnotetext{
1 „uni-deutsch.de“ wird an der Universität München in Kooperation mit der Universität des Saarlandes entwickelt und Mitte 2003 in Betrieb gehen. Es besteht aus zwei Online-Modulen: einem studienvorbe:eitenden und einem studienbegleitenden Modul mit verschiedenen fachsprachlichen Bereichen. Eine Demoversion findet sich unter www.uni-deutsch.de.
} 
die analytische Betrachtung von Software nach Abschluss eines Entwicklungsprojektes. Folgende Evaluationsverfahren können dabei unter anderem zur Anwendung kommen:

- die (mehr oder weniger systematische) Expertenevaluation durch einzelne Gutachter oder Gutachterteams;

- die Abarbeitung von Kriterienlisten und Checklisten;

- die Inhalts- und Medienanalyse;

- die Kosten-Nutzen-Analyse;

- die subjektive Einstellungsmessung durch verschiedene Befragungsverfahren;

- die Verhaltensbeobachtung in Bezug auf Nutzerfreundlichkeit, Lehr-/Lernabläufe etc.;

- die Leistungsmessung, z. B. durch Vergleichsuntersuchungen und Vorher-nachher-Tests.

In dem hier vorgestellten Modell entspricht die retroaktiv-analytische Begutachtung der fertigen Software dem beschriebenen „Expertenverfahren". Der proaktiv-sukzessive Ablauf in Bezug auf die Projektgenese entspricht dem „Entwickler-/Autorenverfahren“. Die drei letztgenannten (und weitere) empirische(n) Evaluationsverfahren bekommen in diesem Modell nicht nur eine bewertende, sondern eine konstruktive und für die Entwicklung konstitutive Rolle zugewiesen. Sie sind dazu gedacht, den Beweis für didaktische Effizienz anzutreten. Das heißt, Sprachlehr-/-lernsoftware kann ohne diese empirischen Beweise nicht erschöpfend bewertet werden.

In einer bisher unveröffentlichten Studie, einer am Multimedia Forschungs- und Entwicklungslabor der Universität München durchgeführten Untersuchung, zur Effizienz von Grammatikanimationen im Rahmen der Entwicklung des „unideutsch.de“-Programms konnten so z. B. neue Einsichten in die Funktion von Bildern beim Spracherwerb gewonnen werden. Dazu wurden zwei Gruppen von insgesamt 30 fortgeschrittenen Lernern des Deutschen als Fremdsprache gebildet, eine Testgruppe und eine Kontrollgruppe. Während die Testgruppe vier verschiedene Grammatikanimationen zur Wortstellung, Kasusmarkierung, Wortbildung und verschiedenen Funktionen des Platzhalters es zu bearbeiten hatte, wurde die Kontrollgruppe in diesen Themen im regulären Sprachunterricht über die jeweilige Lehrkraft unterrichtet. Alle Lerner hatten bereits vor Beginn des Versuchs Sprachunterricht auch zu den genannten Themen erhalten. Hier ging es also um die Wiederholung eigentlich bekannten, aber noch nicht völlig beherrschten Stoffes. Um das jeweilige Eingangsniveau zu ermitteln wurden die Lerner mittels entsprechender Testsätze bewertet. Dann arbeiteten sie selbstständig mit den Grammatikanimationen (Testgruppe) oder erhielten Unterricht (Kontrollgruppe). Messungen der Behaltensleistung wurden unmittelbar im Anschluss an Präsentation oder Unterricht und ein weiteres Mal nach einer Woche vorgenommen. Dabei ergaben sich bei den meisten Testelementen für die Grammatikanimationen deutlichere Verbesserungen der Behaltensleistungen als für die Kontrollgruppe. Diese Verbesserungen sind in Relation zum Eingangsniveau meist signifikant oder annähernd signifikant. Sie lassen Rückschlüsse darauf zu, welche Art von bildlichen Darstellungen (statische oder dynamische) sich am besten für welche grammatischen oder thematischen Eigenschaften und Vermittlungsmethoden anbieten. Im Einzelnen werden Untersuchung und Ergebnisse in Roche/Scheller (2003) dargestellt.

Die Ergebnisse solcher Tests müssen darüber hinaus (wesentlich mehr als bisher) dazu beitragen, dass die theoretischen Annahmen didaktischer Ansätze überprüft und gegebenenfalls modifiziert werden, in der oben skizzierten Untersuchung zum Beispiel in Hinsicht auf die Aussagen einer kognitiven Theorie multimedialen Lernens. Sie werden somit also $\mathrm{zu}$ einem wissenschaftlichen Instrument. In idealer Weise dafür geeignet sind Programmaufzeichnungen des Nutzerverhaltens (z. B. auch zu Lernwegen, Fehlerfrequenzen, Bearbeitungsverhalten, Wiederholungen), da sie von der Software automatisch aufgezeichnet und an die Lehrer/Wissenschaftler weitergeleitet werden können. Hierfür eignen sich naturgemäß besonders Online-Medien.

Nicht nur die Evaluation von Software, sondern die gesamte Sprachlehr-/-lernforschung bekommt durch die hier beschriebene theoretische Orientierung eine neue Qualität. Zur Umsetzung auf ein operationalisierbares $\mathrm{Ni}$ veau bleibt freilich noch ein langer Weg. 


\section{Literatur}

Barkowski, Hans et al. (1980): Deutsch für ausländische Arbeiter. Gutachten zu ausgewählten Lehrwerken. Mainz.

Baumgartner, Peter (1997): Didaktische Anforderungen an (multimediale) Lernsoftware. In: L. J. Issing / P. Klimsa (Hg.), 241-252.

Beers, Maggie (2001): A Media-Based Approach to Developing Ethnographic Skills for Second Language Teaching and Learning. In: Zeitschrift für Interkulturellen Fremdsprachenunterricht 2. $<$ http://www.ualberta.ca/ german/ejournal/beers 2.htm>.

Cognition and Technology Group at Vanderbilt (1992): The Jasper Experiment. An Exploration of Issues in Learning and Instructional Design. In: Educational Technology Research and Development 1, 65-80.

Collins, Allan et al. (1989): Cognitive Apprenticeship. Teaching the Crafts of Reading, Writing, and Mathematics. In: L. B. Resnick (Hg.), Knowing, Learning, and Instruction. Hillsdale, N. J., 453-494.

Doll, Carol A. (1987): Evaluating Educational Software. Chicago/London.

Engel, Ulrich et al. (1979): Mannheimer Gutachten zu ausgewählten Lehrwerken Deutsch als Fremdsprache. Heidelberg.

Fischhaber, Kathrin (2002): Digitale Ethnographie. Eine Methode zum Erlernen interkultureller Kompetenz im Fremdsprachenunterricht. In: Zeitschrift für Interkulturellen Fremdsprachenunterricht 1. <http://www.ualberta.ca/ german/ejournal/ fischhaber1.htm $>$.

Goethe-Institut (Hg.) (1990): Lehrwerkanalyse. Handbuch für die Spracharbeit. München.

Goldman-Segall, Ricky (1998): Points of viewing children's thinking. A digital ethnographer's journey. New Jersey.

Hubbard, Philip (1992): A methodological framework for CALL courseware development. In: D. Sharp et al. (Hg.), Computers in applied linguistics. An international perspective. Clevedon, $39-65$.

Issing, Ludwig J. (1997): Instruktionsdesign für Multimedia. In: L. J. Issing /P. Klimsa (Hg.), 195-220.

Issing, Ludwig J./Klimsa, Paul (Hg.) (1997): Information und Lernen mit Multimedia. Weinheim.

Kast, Bernd/Neuner, Gerhard (Hg.) (1994): Zur Analyse, Begutachtung und Entwicklung von Lehrwerken für den fremdsprachlichen Deutschunterricht. Berlin/München.

Mayer, Richard (1997): Multimedia Learning. Are We Asking the Right Questions? In: Educational Psychologist 4, 1-19.

Mitschian, Haymo (2000): Vom Behaviorismus zum Konstruktivismus. In: Zeitschrift für Interkulturellen Fremdsprachenunterricht $4,3$.
$<$ http://www.ualberta.ca/ german/ejournal/mitschian1.htm $>$.

Murray, Garold L. (1999): Exploring learners' CALL experiences. A reflection on method. In: Computer Assisted Language Learning 12, 179-195.

Papert, Seymour (1980): Mindstorms. Children, computers, and powerful ideas. New York.

Plass, Jan (1998): Design and Evaluation of the User Interface of Foreign Language Multimedia Software. A Cognitive Approach. In: Language Learning \& Technology 1, 35-45.

Reeder, Ken et al. (2001): E/Valuating New Media in Language Development. In: Zeitschrift für Interkulturellen Fremdsprachenunterricht 2/2001. $<$ http://www.ualberta.ca/ german/ejournal/ reeder $1 . h$ tm $>$.

Reinfried, Marcus (1999): Der Radikale Konstruktivismus. Eine sinnvolle Basistheorie für die Fremdsprachendidaktik? In: Fremdsprachen Lehren und Lernen 28, 162-180.

Richards, Jack C./Rodgers, Theodore S. (1986): Approaches and Methods in Language Teaching. Cambridge.

Roche, Jörg (2000): Lerntechnologie und Spracherwerb. Grundrisse einer medienadäquaten, interkulturellen Sprachdidaktik. In: DaF 3, 136-143.

Roche, Jörg (2001): Interkulturelle Sprachdidaktik. Eine Einführung. Tübingen.

Roche, Jörg (2002): Vom Medienmangel in der Lehre und vom Lehrmangel in den Medien. In: V. Schubert (Hg.), Die Geisteswissenschaften in der Informationsgesellschaft. Wissenschaft und Philosophie. St. Ottilien (Interdisziplinäre Studien, 23). Auch unter: <http://werkstadt.daf.unimuenchen.des.

Roche, Jörg/Scheller, Julia (2003, i. Dr.): Zur Effizienz von Grammatikanimationen im Spracherwerb. In: Zeitschrift für Interkulturellen Fremdsprachenunterricht.

Rösler, Dietmar/Tschirner, Erwin (2002): Neue Medien und Deutsch als Fremdsprache. Viele Fragen und ein Aufruf zur Diskussion. In: DaF 3, 144-155.

Rüschoff, Bernd (1999): Wissenskonstruktion als Grundlage fremdsprachlichen Lernens. In: Fremdsprachen Lehren und Lernen 28, 32-43.

Schlickau, Stephan (2001): Praxis und Analyse interkultureller Kommunikation durch Video und Videokonferenz. Lernpotenziale und Anforderungen. In: Zeitschrift für Interkulturellen Fremdsprachenunterricht 2 . <http://www.ualberta.ca/ german/ejournal/schlickau1.htm>.

Spiro, Rand J. et al. (1991): Cognitive Flexibility, Constructivism, and Hypertext. Random Access Instruction for Advanced Knowledge Acquisition in Illstructured Domains. In: Educational Technology 31, 24-33. 
Sprachverband (1994): Beurteilung von Lehrwerken in Kursen, die für ausländische Arbeitnehmer durchgeführt werden. In: B. Kast/G. Neuner (Hg.), 122-130.

Strittmatter, Peter/Mauel, Dirk (1997): Einzelmedium, Medienverbund und Multimedia. In: L. J. Issing / P. Klimsa (Hg.), 48-61.

Tennyson, Robert D. /Christensen, Dean L. (1988): MAIS. An Intelligent Learning System. In: D. H. Jonassen (Hg.), Instructional Designs for Microcomputer Courseware. Hillsdale, N. J., 247-274.

Thomé, Dorothea (1989): Kriterien zur Bewertung von Lernsoftware. Heidelberg.

Tschirner, Erwin (1995): Theorie und Praxis des
Natural Approach in den 90er Jahren. Eine Methode wird volljährig. In: DaF 1, 3-11.

Tschirner, Erwin (1997): Deutsch als Fremdsprache im Medienzeitalter. In: Fremdsprache Deutsch. Sondernummer II, 55-58.

Wendt, Michael (1996): Konstruktivistische Fremdsprachendidaktik. Lerner- und handlungsorientierter Fremdsprachenunterricht aus neuer Sicht. Tübingen.

Wolff, Dieter (1994): Der Konstruktivismus. Ein neues Paradigma in der Fremdsprachendidaktik? In: Die Neueren Sprachen 93, 407-429.

Wolff, Dieter (1996): Kognitionspsychologische Grundlagen neuer Ansätze in der Fremdsprachendidaktik. In: Info DaF 5, 541-560.

\section{Computerprogramme}

A la Rencontre de Philippe (von Gilberte Furstenberg, Yale University Press, New Haven 1993). Anno 1602/Anno 1503 (Max Design 1999/2002).

Berliner sehen (von Ellen Crocker und Kurt Fendt, Yale University Press, New Haven 1999).

Business Challenges (von Nina O'Driscoll and Sarah Macburnie, Addison Wesley Longman 1996).

Dans un Quartier de Paris (von Gilberte Furstenberg und Sabine Levet, Massachusetts Institute of Technology 1997).

Die Fugger II (Sunflowers Interactive 1996).

Edubba. Pilotfassung (von Ken Reeder et al., Lunny Corp., Vancouver 2000).

Einfach Toll (Houghton Mifflin/interactive Factory 1996).

Hollywood Theatrix (Theatrix Interactive, Emeryville 1996).

Reading German. A Multimedia Self-Study Course on German for Special Puposes (5 Bände und CD-ROMs, von Jörg Roche, Canadian Scholars Press International, Toronto 2000).

The Rosetta Stone (Fairfield Language Technologies 1999).

TMX (von Ralf C. Hoffmann, *tmx design 2001).

TUCO (von Heimy F. Taylor, Gessler Educational Software, New York 1987).

uni-deutsch.de (Ludwig-Maximilians-Universität München/Universität des Saarlandes Saarbrücken, München 2003).

WebConstellations (von Ricki Goldmann-Segall, Massachusetts Institute of Technology 1998).

What to say and how to behave in Great Britain (von Larissa Kaminskaya, Software Publishing House Repetitor MultiMedia (Russia) 1999).

Who is Oscar Lake? (Language Publications Interactive 2001). 